\title{
Pravastatin accelerates ischemia-induced angiogenesis through AMP-activated protein kinase
}

\author{
Yasukatsu Izumi $^{1,2}$, Masayuki Shiota ${ }^{1}$, Hiromi Kusakabe ${ }^{1}$, Yuko Hikita ${ }^{1}$, Takafumi Nakao ${ }^{1}$, \\ Yasuhiro Nakamura ${ }^{2}$, Takashi Muro ${ }^{2}$, Katsuyuki Miura ${ }^{3}$, Minoru Yoshiyama $^{2}$ and Hiroshi Iwao ${ }^{1}$
}

Statins exert pleiotropic effects on the cardiovascular system, in part through an increase in nitric oxide (NO) bioavailability. In this study, we examined the role of pravastatin in ischemia-induced angiogenesis. Unilateral hindlimb ischemia was surgically induced in C57BL/6J mice. Phosphorylation of AMP-activated protein kinase (AMPK), acetyl-CoA carboxylase (ACC) and endothelial NO synthase (eNOS) was increased in ischemic tissues. Furthermore, mice treated with pravastatin showed higher increases in phosphorylation than did untreated mice. Laser Doppler analysis has shown that pravastatin treatment accelerates the development of collateral vessels and angiogenesis in response to hindlimb ischemia. Capillary density in the ischemic hindlimb was also increased by pravastatin treatment. An in vitro study on human umbilical vein endothelial cells (HUVECs) revealed that pravastatin increased the phosphorylation of AMPK. Pravastatin-induced phosphorylation of eNOS, one of the downstreams of AMPK, was inhibited by compound C, an AMPK antagonist. The increased migration and tube formation of HUVECs by pravastatin were significantly blocked by compound $C$ treatment. The accelerated angiogenesis by pravastatin after hindlimb ischemia was significantly reduced after treatment with compound C. Thus, ischemia induced AMPK phosphorylation in vivo. Furthermore, pravastatin could also activate AMPK in vivo and in vitro. Such phosphorylation results in eNOS activation and angiogenesis, which provide a novel explanation for one of the pleiotropic effects of statins that is beneficial for angiogenesis.

Hypertension Research (2009) 32, 675-679; doi:10.1038/hr.2009.77; published online 5 June 2009

Keywords: angiogenesis; endothelium; ischemia; nitric oxide synthase; statins

\section{INTRODUCTION}

The AMP-activated protein kinase (AMPK) is a trimeric enzyme comprising a catalytic $\alpha$-subunit and regulatory- $\beta, \gamma$-subunits. AMPK was identified as an upstream kinase that phosphorylates and hence inactivates 3-hydroxy-3-methylglutaryl-coenzyme A reductase and acetyl-coenzyme A carboxylase (ACC), key enzymes that control choresterol/isoprenoid and fatty acid biosynthesis, respectively. AMPK is considered to be a cellular energy sensor that stimulates ATP-producing catabolic pathways and inhibits ATP-consuming anabolic pathways. ${ }^{1}$ Thus, the AMPK pathway is thought to be a regulator of stress responses and cellular energy homeostasis. However, recent studies have shown that AMPK also plays an important role in maintaining endothelial functions. ${ }^{2}$ AMPK activation has beneficial effects on endothelial functions and in antiatherogenesis. These effects include the induction of the endothelial nitric oxide synthase (eNOS) pathway to increase NO bioavailability; the suppression of endothelial reactive oxygen species production when stimulated by hyperglycemia or high free fatty acids to improve endothelial free fatty acid oxidation and limit lipid accumulation; the inhibition of apoptosis and inflammation; and the modulation of the vascular tone. ${ }^{3,4}$
3-Hydroxy-3-methylglutaryl-coenzyme A reductase inhibitors (statins) belong to the class of lipid-lowering medications. Numerous studies, however, suggest that statin therapy has additional cardiovascular protective effects that may function independently of their ability to lower serum cholesterol levels. ${ }^{5,6}$ In addition, the antithrombotic and anti-inflammatory effects of statins have been shown to contribute to the overall beneficial activity of these drugs. ${ }^{7}$ Most importantly, statin therapy improves endothelial function by virtue of its antioxidant and anti-inflammatory effects, ${ }^{8,9}$ as well as by its ability to upregulate eNOS. ${ }^{10}$ The statin activation of Akt has been shown to inhibit apoptosis, acutely increase NO production, induce migration and promote angiogenesis. ${ }^{11}$ These effects of statins were prevented by PI3K inhibitors or by the dominant-negative form of Akt. ${ }^{11}$ Although many studies have reported that these effects depend on eNOS activation by Akt, their action cannot be explained by Akt activation alone. ${ }^{12}$ Indeed, each physiological response downstream of Akt seems to be mediated by multiple targets.

Recent studies have reported that statins can activate AMPK in mouse aorta and myocardium. ${ }^{13}$ Furthermore, AMPK signaling is required for angiogenic responses in endothelial cells (ECs)

\footnotetext{
${ }^{1}$ Department of Pharmacology, Osaka City University Medical School, Osaka, Japan; ${ }^{2}$ Department of Internal Medicine and Cardiology, Osaka City University Medical School, Osaka, Japan and ${ }^{3}$ Applied Pharmacology and Therapeutics, Osaka City University Medical School, Osaka, Japan 
under hypoxic conditions in vitro. ${ }^{14}$ In addition, adiponectin or 5aminoimidazole-4-carboxamide-1- $\beta$-D-ribofuranoside (AICAR) and AMPK activators promote angiogenesis in response to ischemic stress. ${ }^{15,16}$ Furthermore, atrovastatin-induced eNOS phosphorylation was attenuated in the aorta of AMPK $\alpha 2^{-1-}$ mice. ${ }^{17}$ Thus, eNOS phosphorylation may be at least partly mediated by AMPK activation. However, the molecular mechanism of angiogenesis by statins is not fully understood. Here, we investigated the role of AMPK in statininduced eNOS phosphorylation in vitro and in vivo. Our results show that pravastatin can activate AMPK in an ischemic hindlimb, with the assistance of eNOS.

\section{METHODS}

\section{Materials}

Pravastatin was provided as a gift from Daiichi Sankyo Co. Ltd. (Tokyo, Japan). The antibodies used in this study and their commercial sources were as follows: anti-phospho-AMPK (p-Thr172), anti-AMPK, anti-phospho-Akt (p-Ser473), anti-Akt, anti-phospho-ACC (p-Ser79), anti-ACC and anti-phospho-eNOS (p-Ser1177) (Cell Signaling Technology, Beverly, MA, USA); antieNOS (BD Biosciences, San Jose, CA, USA); anti- $\beta$-actin (SIGMA, St Louis, MO, USA); and the horseradish-peroxidase-coupled secondary antibodies (GE Healthcare, Chalfont St Giles, UK). Compound C, an AMPK inhibitor, was purchased from Calbiochem (San Diego, CA, USA). Matrigel was obtained from BD Biosciences.

\section{Animal experiments}

All procedures were in accordance with the institutional guidelines for animal research. All experiments were performed using male C57BL/6J mice aged 8-9 weeks (Japan CREA, Tokyo, Japan). Unilateral hindlimb ischemia was induced by resecting the left femoral arteries and veins of mice under anesthesia with sodium pentobarbital ( $50 \mathrm{mg} \mathrm{kg}^{-1}$ intraperitoneally), as described previously. ${ }^{18}$

In the first experiment, we examined the time course of ischemia-induced AMPK, eNOS and Akt activities. Next, the mice were fed with pravastatin at $20 \mathrm{mg} \mathrm{kg}^{-1}$ body weight by gastric gavage. Saline was fed to control animals as control. After $4,8,16$ or $24 \mathrm{~h}$, the mice were killed and their hindlimbs were removed and stored in $-80{ }^{\circ} \mathrm{C}$ for western blotting. Furthermore, hindlimb ischemia was induced after $7 \mathrm{~h}$ of pravastatin administration by gastric gavage, and the mice were killed after $60 \mathrm{~min}$ of ischemia induction.

In the second experiment, to investigate the effect of pravastatin on hindlimb blood flow, the mice were administered pravastatin $\left(20 \mathrm{mg} \mathrm{kg}^{-1} \mathrm{day}^{-1}\right)$ in drinking water from the day of ischemia induction.

\section{Laser Doppler blood flow analysis}

Hindlimb blood flow was measured using a laser Doppler blood flow (LDBF) analyzer (Moor LDI; Moor Instruments, Axminster, UK) as described previously. ${ }^{18}$ Before the operation and on postoperative days 4, 7, 14, 21 and 28, we performed LDBF analysis on the legs and feet. After scanning the blood flow twice, the stored images were subjected to computer-assisted quantification, and the average flows of the ischemic and non-ischemic limbs were calculated. To avoid data variations due to ambient light and temperature, hindlimb blood flow was expressed as the ratio of left (ischemic) to right (non-ischemic) LDBF.

\section{Capillary density}

Capillary density within the ischemic thigh adductor skeletal muscles was analyzed to obtain a specific evidence of vascularity at the microcirculation level, similar to our previous method. ${ }^{18}$ Three pieces of ischemic muscles were harvested from each animal, sliced and fixed in methanol. The tissues were embedded in paraffin, and multiple tissue slices of $5 \mu \mathrm{m}$ thickness were prepared. Capillary ECs were identified by immunohistochemical staining with a rat anti-mouse $\mathrm{CD} 31$ antibody (Ab) (BD Biosciences). Fifteen random microscopic fields from three different sections in each tissue block were examined for the presence of capillary ECs, and capillary density was expressed as the number of capillaries per high-power field $(\times 400)$.

\section{Preparation of extracts and western blot analysis}

Our detailed method has been previously described. ${ }^{18,19}$ Protein extracts were obtained from homogenized ischemic or non-ischemic skeletal muscles. After an electrophoretic transfer to polyvinylidine difluoride membranes (Immobilon-P, Millipore, Billerica, MA, USA), the membranes were probed with each primary antibody.

\section{Effects of AMPK inhibitor on ischemia-induced angiogenesis}

Mice were injected intraperitoneally with $10 \mathrm{mg} \mathrm{kg}^{-1}$ of compound C dissolved in DMSO (Wako Chemicals, Osaka, Japan) or with DMSO alone before inducing ischemia thrice weekly. We measured hindlimb blood flow until day 28 , as mentioned above.

\section{Cell culture, migration and tube formation}

EGM2 and EBM2, culture media and human umbilical vein ECs (HUVECs, passage 3-5) were purchased from Lonza (Basel, Switzerland). The cells were cultured at $37^{\circ} \mathrm{C}$, in $5 \% \mathrm{CO}_{2}$ in EGM2 medium. Three $\mu \mathrm{moll} \mathrm{l}^{-1}$ of compound $\mathrm{C}$ was treated before $30 \mathrm{~min}$ of pravastatin stimulation. All experiments were performed at least four times repeatedly.

The cells were washed with phosphate-buffered saline and lysed in RIPA buffer containing $50 \mathrm{mmoll}^{-1}$ HEPES ( $\mathrm{pH} 8.0$ ), $150 \mathrm{mmoll}^{-1} \mathrm{NaCl}$, $5 \mathrm{mmoll}^{-1}$ EDTA, $1 \%$ CHAPS, $10 \%$ glycerol, $100 \mathrm{mmoll}^{-1} \mathrm{NaF}, 0.2 \mathrm{mmoll}^{-1}$ DTT, $1 \mathrm{mmoll}^{-1}$ phenylmethylsulfonyl fluoride, protease inhibitor cocktail (Nacalai Tesque, Osaka, Japan) for western blotting.

Transwell inserts (Nalgen Nunc, Rochester, NY, USA) were coated with $0.1 \mathrm{mg} \mathrm{ml}^{-1}$ collagen. Pravastatin $\left(1 \mu \mathrm{moll}^{-1}\right)$ dissolved in EBM-2 medium containing $0.25 \%$ bovine serum albumin was added to the bottom chamber of the Boyden apparatus. HUVECs $\left(1 \times 10^{5}\right.$ cells $)$ suspended in a $100 \mu \mathrm{l}$ aliquot of EBM-2 containing $0.25 \%$ bovine serum albumin were added to the upper chamber. After $5 \mathrm{~h}$ incubation, the non-migrating cells in the upper part of the chamber were removed mechanically, and the remaining cells on the lower part were fixed with methanol. The migrated cells were counted in six random microscopic fields after staining with Diff-Quik solutions (Sysmex International Reagents Co. Ltd, Kobe, Japan).

The formation of vascular-like structures by HUVECs was assessed on growth factor-reduced Matrigel (BD Biosciences). Cells were plated at $1 \times 10^{4}$ well $^{-1}$ in a 96-well-multidish, precoated with $30 \mathrm{ml}$ of Matrigel in the absence or presence of pravastatin $\left(1 \mu \mathrm{moll}^{-1}\right)$. After $5 \mathrm{~h}$, the length of the completed tube-like structure in the center field (magnification, $\times 10$ ) was quantified. Images were captured with a CCD color camera attached to the microscope and the tube length was measured using a Micro Analyzer (JPD, Tokyo, Japan).

\section{Statistical analysis}

All data are presented as mean \pm s.e.m. Comparisons among groups were made by one-way analysis of variance. For differences between two groups, Student's $t$-test was used when appropriate. Differences were considered statistically significant at a value of $P<0.05$.

\section{RESULTS}

Time course of AMPK activation after ischemia and pravastatin treatment

All mice survived after the surgical induction of unilateral hindlimb ischemia. We investigated AMPK, Akt and eNOS activities in ischemic hindlimb. As shown in Figure 1a, AMPK was remarkably activated at $15 \mathrm{~min}$ after hindlimb ischemia and peaked at $60 \mathrm{~min}$. Akt and eNOS activities were also increased in response to ischemia. The level of phosphorylated AMPK in the hindlimb increased $4 \mathrm{~h}$ after pravastatin administration and lasted for at least $24 \mathrm{~h}$ (Figure $1 \mathrm{~b}$ ). The phosphorylation of ACC, Akt and eNOS was elevated. Therefore, we next examined the effect of pravastatin on the phosphorylation of AMPK, ACC, Akt and eNOS in the ischemic tissue. AMPK phosphorylation after $60 \mathrm{~min}$ of ischemia induction was additionally increased with pravastatin administration (Figure 1c). The level of AMPK and $\beta$-actin was not changed. 
Unilateral hindlimb ischemia and time course of LDBF analysis Immediately after the left femoral artery and vein were resected, the ratio of ischemic (left) to non-ischemic (right) hindlimb LDBF (the LDBF ratio) decreased from $1.06 \pm 0.05$ to $0.08 \pm 0.01$ without pravastatin and from $1.00 \pm 0.01$ to $0.10 \pm 0.01$ with pravastatin. Thus, the severity of induced ischemia was comparable in the two groups.

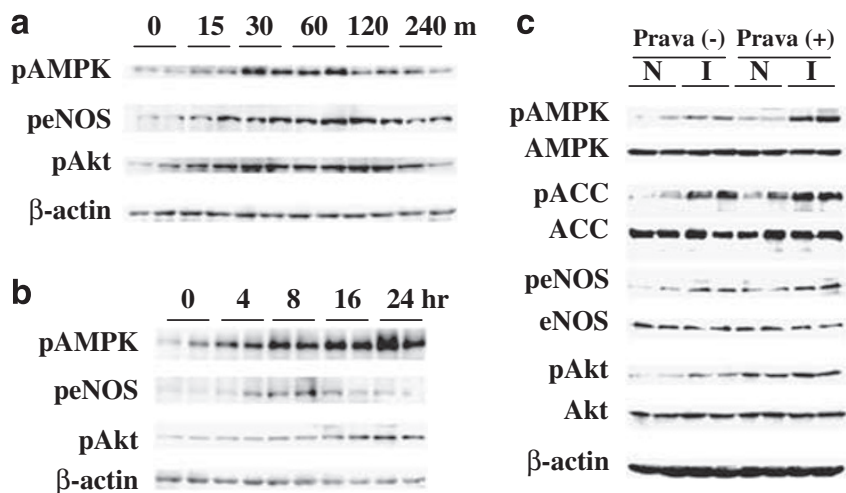

Figure 1 Time course of ischemia-induced AMPK, Akt, ACC and eNOS activation in mice. (a) Representative western blot analysis of these activations at $0,15,30,60,120$ and $240 \mathrm{~min}$ after ischemia. (b) Representative western blot analysis of AMPK, Akt and eNOS phosphorylation at $0,4,8,16$ and $24 \mathrm{~h}$ after pravastatin treatment. (c) Representative western blot analysis of AMPK, ACC, eNOS and Akt. AMPK phosphorylation after $60 \mathrm{~min}$ of ischemia induction was further increased after pravastatin administration (after $8 \mathrm{~h}$ ). pAMPK, phosphoAMPK; pAkt, phospho-Akt; peNOS, phospho-eNOS; pACC, phospho-ACC; Prava, pravastatin; N, non-ischemic muscle; I, ischemic muscle.
Figure 2a shows the representative LDBF images of hindlimb blood flow. A serial LDBF examination disclosed a progressive recovery of hindlimb blood flow in the pravastatin-untreated mice after ischemia was induced. On the other hand, the blood flow of mice treated with pravastatin was accelerated during the follow-up period. Compared with control mice, the LDBF ratio in the pravastatin-treated mice was persistently high after 4 days following the induction of ischemia, and significantly increased except on day $14(P<0.05)$ (Figure $2 b)$.

\section{Tissue capillary density}

To investigate the extent of angiogenesis at the microcirculation level, we measured capillary density in histological sections harvested from ischemic tissues. Quantitative analysis revealed that the capillary density was significantly augmented by pravastatin on postoperative day 28 , compared with that in control mice (Figure 2c).

\section{Effects of compound C on pravastatin-induced eNOS} phosphorylation, migration and tube formation in HUVECs To determine whether AMPK activation is directly concerned with endothelial functions, we studied the effects of compound $\mathrm{C}$, an AMPK inhibitor, on angiogenesis. Angiogenesis incorporates migration, intercellular connections with other ECs and lumen formation in vitro. Angiogenesis was assessed by tube formation assay using Matrigel. Compound C $\left(3 \mu \mathrm{moll}^{-1}\right)$ significantly inhibited pravastatin-induced tube formation (Figure 3a). As angiogenesis involves cell migration, we investigated the role of compound $\mathrm{C}$ in the pravastatininduced migration of HUVECs, using a modified Boyden chamber method. Compound $\mathrm{C}$ treatment inhibited pravastatin-induced cell migration (Figure 3b).
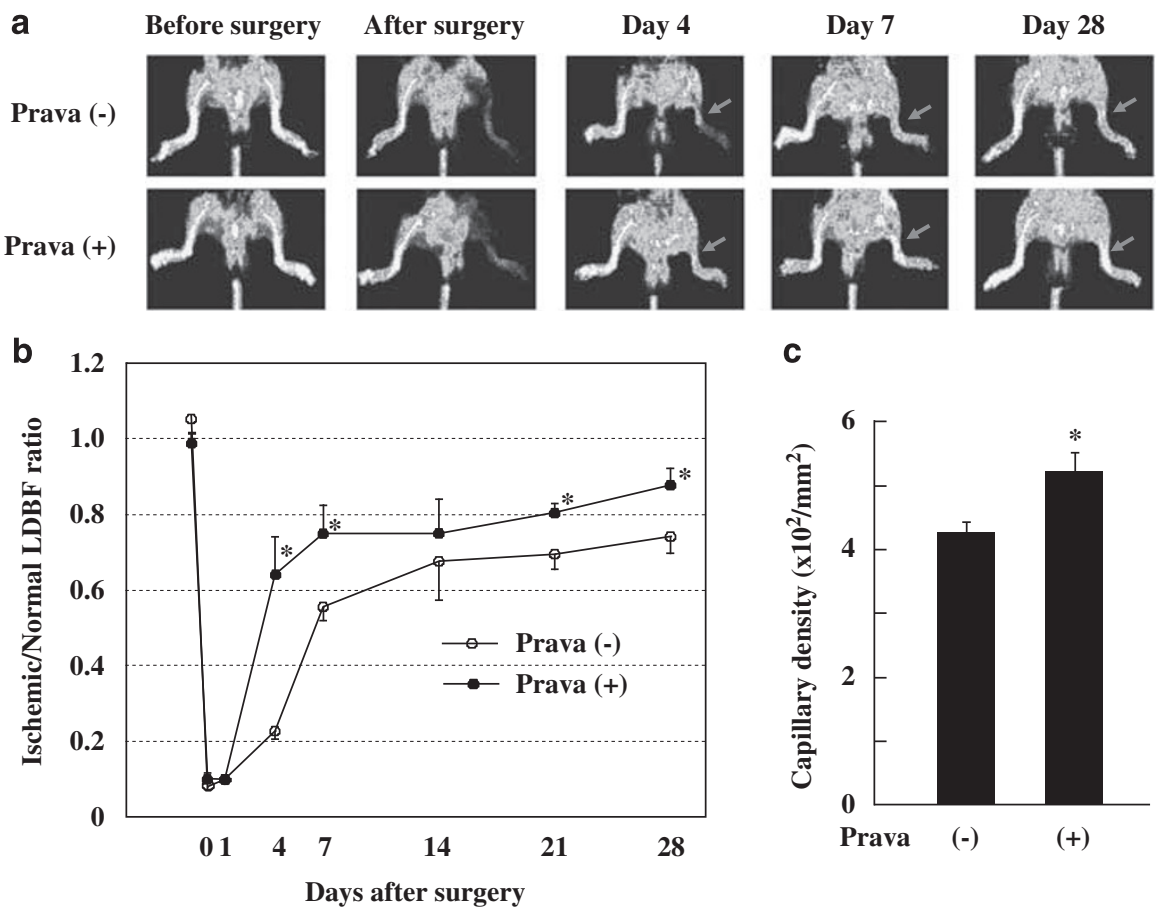

C

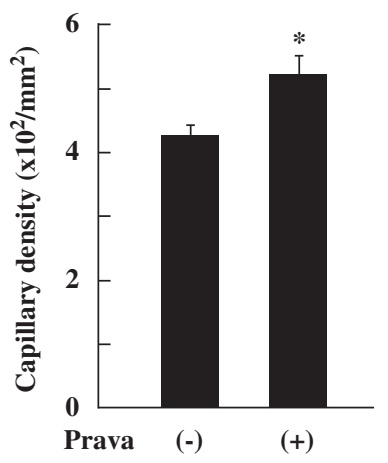

Figure 2 Laser Doppler blood flow (LDBF) analysis and capillary density. (a) Representative LDBF image. A low-perfusion signal was observed in the ischemic hindlimb, whereas a high-perfusion pattern (white to red) was detected in pravastatin-treated mice. (b) Computer-assisted quantitative analysis of hindlimb blood perfusion showed a significant improvement in the ischemic/normal hindlimb blood flow ratio in pravastatin-treated mice ( $n=6)$ compared with that in the untreated animals $(n=6)$. Each bar represents mean \pm s.e.m. ${ }^{*} P<0.01$ vs. Prava $(-)$ at each time point. (c) Capillary density assessed by staining with anti-CD31 Ab on postoperative day 28 ( $n=6$ in each group). pAMPK, phospho-AMPK; pAkt, phospho-Akt; peNOS, phospho-eNOS; pACC, phospho-ACC; Prava, pravastatin; N, non-ischemic muscle; I, ischemic muscle. Each bar represents mean \pm s.e.m. ${ }^{*} P<0.01$ vs. Prava (-). A full color version of this figure is available at the Hypertension Research journal online. 


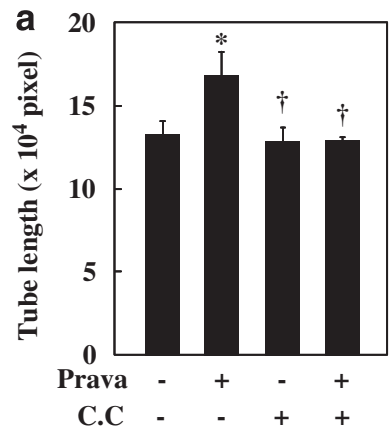

C

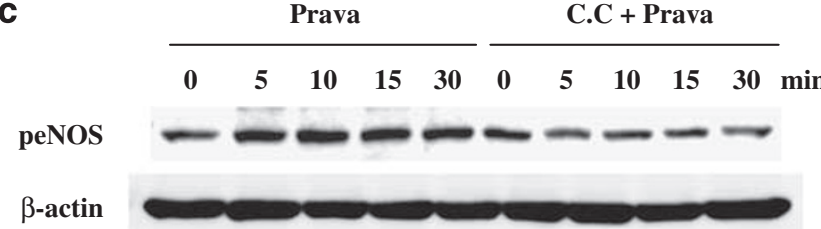

Figure 3 Effects of compound $C$ on pravastatin-induced tube formation (a), migration (b), and eNOS phoshorylation (c) in HUVECs. C.C, compound C; pAMPK, phospho-AMPK; pAkt, phospho-Akt; peNOS, phospho-eNOS; pACC, phospho-ACC; Prava, pravastatin; N, non-ischemic muscle; I, ischemic muscle. Each bar represents mean \pm s.e.m. $(n=4) .{ }^{*} P<0.01$ vs. Prava $(-) / C . C(-),{ }^{\dagger} P<0.01$ vs. Prava $(+) / C . C(-)$. Experiments were repeated three times and similar results were obtained.

As eNOS activation is important in endothelial function, we next measured the phosphorylation of eNOS. Pravastatin $\left(1 \mu \mathrm{moll}^{-1}\right)$ induced the phosphorylation of eNOS at Ser1177, which could be blocked by compound C pretreatment for $30 \mathrm{~min}$ (Figure 3c). Taken together, these results suggest that blockade of AMPK suppressed angiogenesis, which indicates that endogenous AMPK is important for the angiogenic activity of ECs.

\section{In vivo blockade of AMPK weakens angiogenesis}

We finally examined the effect of compound $\mathrm{C}$ on pravastatin-induced angiogenesis in vivo. The recovery of hindlimb blood flow in mice treated with compound $\mathrm{C}$ was weaker than that in control (DMSO)treated mice after 14 days following the induction of ischemia. The increased LDBF ratio by pravastatin administration was also reduced with compound C (Figure 4a). On postoperative day 28 , the increase in capillary density by pravastatin was also reduced with the treatment of compound C (Figure $4 \mathrm{~b}$ ).

\section{DISCUSSION}

In this study, angiogenesis and blood flow recovery in response to hindlimb ischemia were significantly advanced by pravastatin, and increased angiogenesis was blocked by compound C. Thus, pravastatin displays proangiogenic effects through AMPK-eNOS activation on ECs.

Recent studies have suggested that AMPK is necessary for hypoxiainduced VEGF mRNA stabilization, and regulates VEGF expression and capillarization. ${ }^{20,21}$ Furthermore, recent reports state that statins reduce cardiovascular events and enhance ischemia-induced angiogenesis. ${ }^{11,12,22}$ In this study, we investigated whether the statin activation of AMPK involved angiogenesis.

We used pravastatin to demonstrate the positive effect of statins on the phosphorylation of AMPK (Thr172) in ECs. Such phosphorylation is essential for AMPK activation, which is also demonstrated by increased AMPK activity and increased phosphorylation of ACC

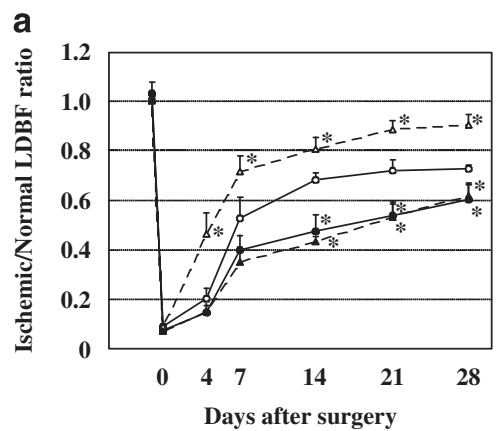

b

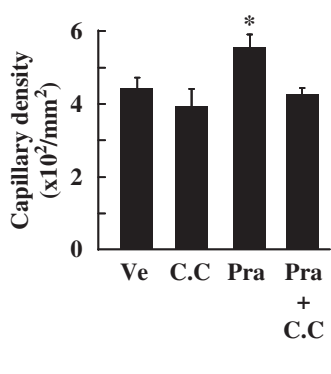

Figure 4 Effects of compound $\mathrm{C}$ on angiogenesis. (a) Computer-assisted quantitative analysis of hindlimb blood perfusion by LDBF analyzer. (b) Capillary density on postoperative day $28 . \bigcirc$, vehicle; $\mathbf{O}$, compound $\mathrm{C}$; $\triangle$, pravastatin; $\boldsymbol{\Lambda}$, pravastatin and compound C; C.C, compound C; PAMPK, phospho-AMPK; pAkt, phospho-Akt; peNOS, phospho-eNOS; pACC, phospho-ACC; Prava, pravastatin; N, non-ischemic muscle; I, ischemic muscle. Each bar represents mean \pm s.e.m. $(n=5-6) .{ }^{*} P<0.05$ vs. vehicle.

(Ser79), a direct target of AMPK. ${ }^{23}$ Other statins such as atrovastatin and lovastatin can also cause AMPK and ACC phosphorylation in ECs. ${ }^{13}$ Furthermore, Xenos et al. ${ }^{24}$ showed that the level of AMPK protein in human ECs increased following $48 \mathrm{~h}$ of fluvastatin treatment. On the other hand, AMPK phosphorylation in ECs was increased by statins in a rapid and transient manner. This temporal response is similar to that stimulated by shear stress, by adiponectin and by metformin. ${ }^{25-28}$ Sun et al. ${ }^{13}$ showed that the phosphorylation/ activation of AMPK in mouse aorta and myocardium was observed as early as $2 \mathrm{~h}$ and peaked at $4-8 \mathrm{~h}$ after atrovastatin administration. However, the role of AMPK in angiogenesis in vivo has not been fully elucidated. The present data indicate that AMPK was transiently phosphorylated by ischemia (Figure 1a). Furthermore, the phosphorylation of AMPK in the mouse hindlimb was observed as early as $4 \mathrm{~h}$ and peaked at $16-24 \mathrm{~h}$ after pravastatin administration (Figure $1 \mathrm{~b}$ ). From this finding and from the report stating that activated AMPK in muscle induces VEGF production and promotes angiogenesis, ${ }^{16}$ the tissues in which AMPK activation was observed in the hindlimb in vivo were the endothelium and mycocytes.

The effect of statins on eNOS activation in ECs is dependent on the phosphatidylinositol-3 kinase (PI3K)-Akt pathway. ${ }^{11,29}$ Our data indicate that AMPK is also engaged in the upregulation of eNOS by pravastatin. The association between Akt and AMPK in eNOS phosphorylation (Ser 1177) is elusive. ${ }^{30}$ Dimmeler et al. ${ }^{29}$ have shown that shear stress-induced eNOS phosphorylation was blocked by a PI3K inhibitor, wortmannin, in ECs. On the other hand, Chen et al. ${ }^{25}$ have shown that a dominant-negative mutant of AMPK (DN-AMPK), but not of Akt, significantly inhibited eNOS phosphorylation and NO production in ECs in response to adiponectin. Very recently, Sun et al. ${ }^{13}$ have shown that eNOS phosphorylation in HUVECs was inhibited by DN-AMPK or by compound C, whereas Akt phosphorylation was drastically increased by the downregulation of AMPK. Our present data support the hypothesis that eNOS activation depends not only on Akt but also on AMPK, because inhibition of AMPK attenuated statin-activated eNOS. A recent study ${ }^{17}$ that atrovastatin-induced eNOS phosphorylation was attenuated in AMPK $\alpha 2^{-I-}$ mice supports our results. On the other hand, Ouchi et al. ${ }^{26}$ have shown that DN-Akt blocked adiponectin-stimulated Akt and eNOS phosphorylation without altering AMPK phosphorylation, whereas DN-AMPK inhibited adiponectin-induced Akt phosphorylation. These results suggest that AMPK may be upstream of Akt. 
The AMP-activated protein kinase seems to play a central role in metabolic regulations as its activation improves metabolic abnormalities that are found in diabetic mice and rats. ${ }^{31,32}$ Transgenic mice expressing a kinase-dead mutant of AMPK showed increased apoptosis and cardiac dysfunction after an ischemia-reperfusion injury. ${ }^{33}$ Furthermore, adiponectin has been shown to protect the heart from ischemia-reperfusion injury through the AMPK pathway. ${ }^{34}$ We found that compound C significantly decreased pravastatin-induced eNOS phosphorylation in HUVECs, thereby confirming that pravastatin regulates eNOS activity through AMPK. Thus, the pharmacological activators of AMPK signaling could produce beneficial effects. In addition, the effects of statin-activated AMPK could extend beyond the protection of the vascular endothelium.

In conclusion, our present work has provided the first in vivo evidence that angiogenesis and blood flow recovery in response to hindlimb ischemia are accelerated by pravastatin and that the increased angiogenesis is blocked by compound C. Thus, pravastatin will have proangiogenic effects through AMPK-eNOS activation on ECs. These findings suggest that AMPK could be a new therapeutic target for vascular diseases.

\section{CONFLICT OF INTEREST}

The authors declare no conflict of interest.

\section{ACKNOWLEDGEMENTS}

We thank Ms Azusa Inagaki and Ms Masako Tanaka for their technical assistance. This study was supported in part by a Grant-in-Aid for Scientific Research from the Ministry of Education, Culture, Sports, Science and Technology of Japan (20590257).

1 Kahn BB, Alquier T, Carling D, Hardie DG. AMP-activated protein kinase: ancient energy gauge provides clues to modern understanding of metabolism. Cell Metab 2005; 1: 15-25.

2 Zou MH, Wu Y. AMP-activated protein kinase activation as a strategy for protecting vascular endothelial function. Clin Exp Pharmacol Physiol 2008; 35: 535-545.

3 Hattori Y, Suzuki K, Hattori S, Kasai K. Metformin inhibits cytokine-induced nuclear factor kappaB activation via AMP-activated protein kinase activation in vascular endothelial cells. Hypertension 2006; 47: 1183-1188.

4 Kobayashi H, Ouchi N, Kihara S, Walsh K, Kumada M, Abe Y, Funahashi T, Matsuzawa Y. Selective suppression of endothelial cell apoptosis by the high molecular weight form of adiponectin. Circ Res 2004; 94: e27-e31.

5 West of Scotland Coronary Prevention Study Group. Influence of pravastatin and plasma lipids on clinical events in the West of Scotland Coronary Prevention Study (WOSCOPS). Circulation 1998; 97: 1440-1445.

6 Liao JK. Clinical implications for statin pleiotropy. Curr Opin Lipidol 2005; 16 : 624-629.

7 Vaughan CJ, Delanty N. Neuroprotective properties of statins in cerebral ischemia and stroke. Stroke 1999; 30: 1969-1973.

8 Cahoon Jr WD, Crouch MA. Preprocedural statin therapy in percutaneous coronary intervention. Ann Pharmacother 2007; 41: 1687-1693.

9 Dilaveris P, Giannopoulos G, Riga M, Synetos A, Stefanadis C. Beneficial effects of statins on endothelial dysfunction and vascular stiffness. Curr Vasc Pharmacol 2007; 5: 227-237.

10 Laufs U, Endres M, Stagliano N, Amin-Hanjani S, Chui DS, Yang SX, Simoncini T, Yamada M, Rabkin E, Allen PG, Huang PL, Bohm M, Schoen FJ, Moskowitz MA, Liao JK. Neuroprotection mediated by changes in the endothelial actin cytoskeleton. J Clin Invest 2000; 106: 15-24.

11 Kureishi Y, Luo Z, Shiojima I, Bialik A, Fulton D, Lefer DJ, Sessa WC, Walsh K. The HMG-CoA reductase inhibitor simvastatin activates the protein kinase Akt and promotes angiogenesis in normocholesterolemic animals. Nat Med 2000; 6: 1004-1010.
12 Sata M, Nishimatsu H, Suzuki E, Sugiura S, Yoshizumi M, Ouchi Y, Hirata Y, Nagai R. Endothelial nitric oxide synthase is essential for the HMG-CoA reductase inhibitor cerivastatin to promote collateral growth in response to ischemia. FASEB J 2001; 15: 2530-2532.

13 Sun W, Lee TS, Zhu M, Gu C, Wang Y, Zhu Y, Shyy JY. Statins activate AMP-activated protein kinase in vitro and in vivo. Circulation 2006; 114: 2655-2662.

14 Nagata D, Mogi M, Walsh K. AMP-activated protein kinase (AMPK) signaling in endothelial cells is essential for angiogenesis in response to hypoxic stress. $J \mathrm{Biol}$ Chem 2003; 278: 31000-31006.

15 Shibata R, Ouchi N, Kihara S, Sato K, Funahashi T, Walsh K. Adiponectin stimulates angiogenesis in response to tissue ischemia through stimulation of AMP-activated protein kinase signaling. J Biol Chem 2004; 279: 28670-28674.

16 Ouchi N, Shibata R, Walsh K. AMP-activated protein kinase signaling stimulates VEGF expression and angiogenesis in skeletal muscle. Circ Res 2005; 96: 838-846.

17 Chen Z, Peng IC, Sun W, Su MI, Hsu PH, Fu Y, Zhu Y, DeFea K, Pan S, Tsai MD, Shyy JY. AMP-activated protein kinase functionally phosphorylates endothelial nitric oxide synthase Ser633. Circ Res 2009; 104: 496-505.

18 Izumi Y, Kim-Mitsuyama S, Yoshiyama M, Omura T, Shiota M, Matsuzawa A, Yukimura T, Murohara T, Takeya M, Ichijo H, Yoshikawa J, Iwao H. Important role of apoptosis signal-regulating kinase 1 in ischemia-induced angiogenesis. Arterioscler Thromb Vasc Biol 2005; 25: 1877-1883.

19 Izumi Y, Kim S, Yoshiyama M, Izumiya Y, Yoshida K, Matsuzawa A, Koyama H, Nishizawa $\mathrm{Y}$, Ichijo $\mathrm{H}$, Yoshikawa J, Iwao $\mathrm{H}$. Activation of apoptosis signal-regulating kinase 1 in injured artery and its critical role in neointimal hyperplasia. Circulation 2003; 108: 2812-2818.

20 Lee M, Hwang JT, Yun H, Kim EJ, Kim MJ, Kim SS, Ha J. Critical roles of AMPactivated protein kinase in the carcinogenic metal-induced expression of VEGF and HIF-1 proteins in DU145 prostate carcinoma. Biochem Pharmacol 2006; 72: 91-103.

21 Zwetsloot KA, Westerkamp LM, Holmes BF, Gavin TP. AMPK regulates basal skeletal muscle capillarization and VEGF expression, but is not necessary for the angiogenic response to exercise. J Physiol 2008; 586: 6021-6035.

22 Llevadot J, Murasawa S, Kureishi Y, Uchida S, Masuda H, Kawamoto A, Walsh K, Isner JM, Asahara T. HMG-CoA reductase inhibitor mobilizes bone marrow-derived endothelial progenitor cells. J Clin Invest 2001; 108: 399-405.

23 Stein SC, Woods A, Jones NA, Davison MD, Carling D. The regulation of AMP-activated protein kinase by phosphorylation. Biochem J 2000; 345 (Part 3): 437-443.

24 Xenos ES, Stevens SL, Freeman MB, Cassada DC, Goldman MH. Nitric oxide mediates the effect of fluvastatin on intercellular adhesion molecule- 1 and platelet endothelial cell adhesion molecule-1 expression on human endothelial cells. Ann Vasc Surg 2005; 19: 386-392.

25 Chen H, Montagnani M, Funahashi T, Shimomura I, Quon MJ. Adiponectin stimulates production of nitric oxide in vascular endothelial cells. J Biol Chem 2003; 278: $45021-45026$.

26 Ouchi N, Kobayashi H, Kihara S, Kumada M, Sato K, Inoue T, Funahashi T, Walsh K. Adiponectin stimulates angiogenesis by promoting cross-talk between AMP-activated protein kinase and Akt signaling in endothelial cells. J Biol Chem 2004; 279 : 1304-1309.

27 Schulz E, Anter E, Zou MH, Keaney Jr JF. Estradiol-mediated endothelial nitric oxide synthase association with heat shock protein 90 requires adenosine monophosphatedependent protein kinase. Circulation 2005; 111: 3473-3480.

28 Zhang Y, Lee TS, Kolb EM, Sun K, Lu X, Sladek FM, Kassab GS, Garland Jr T, Shyy JY AMP-activated protein kinase is involved in endothelial NO synthase activation in response to shear stress. Arterioscler Thromb Vasc Biol 2006; 26: 1281-1287.

29 Dimmeler S, Fleming I, Fisslthaler B, Hermann C, Busse R, Zeiher AM. Activation of nitric oxide synthase in endothelial cells by Akt-dependent phosphorylation. Nature 1999; 399: 601-605.

30 Sessa WC. eNOS at a glance. J Cell Sci 2004; 117: 2427-2429.

31 Fiedler M, Zierath JR, Selen G, Wallberg-Henriksson H, Liang Y, Sakariassen KS. 5-aminoimidazole-4-carboxy-amide-1-beta-D-ribofuranoside treatment ameliorates hyperglycaemia and hyperinsulinaemia but not dyslipidaemia in KKAy-CETP mice. Diabetologia 2001; 44: 2180-2186.

32 Hardie DG. Minireview: the AMP-activated protein kinase cascade: the key sensor of cellular energy status. Endocrinology 2003; 144: 5179-5183.

33 Russell III RR, Li J, Coven DL, Pypaert M, Zechner C, Palmeri M, Giordano FJ, Mu J, Birnbaum MJ, Young LH. AMP-activated protein kinase mediates ischemic glucose uptake and prevents postischemic cardiac dysfunction, apoptosis, and injury. J Clin Invest 2004; 114: 495-503.

34 Shibata R, Sato K, Pimentel DR, Takemura Y, Kihara S, Ohashi K, Funahashi T, Ouchi N, Walsh K. Adiponectin protects against myocardial ischemia-reperfusion injury through AMPK- and COX-2-dependent mechanisms. Nat Med 2005; 11: 1096-1103. 